\title{
Higher Order Statistical Approach for Performance Evaluation of different Spectrum Sensing Techniques in Cognitive Radio Network
}

\author{
Navjot Singh \\ Department of Computer Science \& Engineering, \\ Shri Guru Granth Sahib World University, \\ Fatehgarh Sahib, India
}

\author{
Amandeep Kaur \\ Asst. Prof. \\ Department of Computer Science \& Engineering, \\ Shri Guru Granth Sahib World University, \\ Fatehgarh Sahib, India
}

\begin{abstract}
Cognitive radio is an intelligent wireless technology that increases the spectrum efficiency for its usage in applications. $\mathrm{CR}$ enriches wireless technology by utilizing the spectrum holes in order to provide high order quality service to users and to minimize the interference that can occur in the network. The work that has to be proposed we will uses two Spectrum Sensing techniques for Cognitive radio network which include Cyclostationary detection and Energy detection techniques. In this paper, the parameter used for Cyclostationary signal is Spectral Correlation function(SCF). The detection capability of this (SCF) with different windows is used to check the periodicity of the signal using different-different windows. Due to the periodicity of the baseband signal, SCF would be able to detect the primary user signal at very low SNR. We also analyzed in our proposed work that capability of periodicity of the signal of SCF is not only limited to noise affected signal, it also able to detect the attenuated signal. We have also simulated Energy detection over MIMO fading channel as it models both Rician fading channel and Rayleigh fading channel. The performance in terms of Bit error rate by providing low probability of false alarm and high probability of detection is analyzed. The Statistical test based comparison is made between the two sensing techniques to evaluate the performance in terms of signal to noise ratio. In the proposed work An extensive set of simulations have been conducted in MATLAB
\end{abstract}

\section{Keywords}

Cognitive radio, Energy Detection, Cyclostationary Detection

\section{INTRODUCTION}

The idea of Cognitive radio was first conceived by Sir Joseph Mitola at the Royal Institute of Technology in year 1998. The main factor behind this was increasing need for the radio spectrum [1]. The improved communication speed was the unusual drive and this was satisfied by effectively utilizing the radio spectrum. Due to static allocation of spectrum, the allotted spectrum was not properly utilized. It has become most difficult to find vacant bands either to set up a new service or to enhance the existing one. In order to overcome these problems we are going for "Dynamic Spectrum Management" which also improves the utilization of spectrum. Cognitive Radio performs on this dynamic Spectrum Management principle which solves the issue of spectrum underutilization in wireless communication in an efficient manner [2]. The unlicensed systems(Secondary users) are allowed to use unused spectrum of licensed systems (Primary users). Thus in order to check the band occupancy, unlicensed user should have Cognitive radio capabilities such as sensing to exploit the unused bands of the spectrum [3]. Sensing means to identify free frequency band in the radio environment and plays an important role in $\mathrm{CR}$. The main task of CR is to detect the existence of the primary user by secondary user and to leave the frequency band if the corresponding primary radio emerges in order to prevent interferences to primary users In this paper, Energy detection and Cyclostationary spectrum sensing techniques have been used [4]. While the Energy detection is less complex as compared to cyclostationary its performance degrades at low signal to noise ratio or with noise uncertainty. Cylostationary on the other hand provides effective detection but is computationally more complex and requires longer observation time. In the existing literature, the parameter used for cyclostationary signal is Spectral Correlation function. We exploit the detection capability of SCF with different windows to check the periodicity of the signal using different windows which include Hamming window, Kaiser Window, Rectangular window. It is recorded that capability of periodicity of the signal of SCF is not only limited to noise affected signal, it is also able to detect the attenuated signal. We also simulated Energy detector over MIMO fading channel as it models both Rician, Rayleigh fading channel. We analyzed the result of energy detector in terms of probability of detection providing high probability of detection and low probability of false alarm. The performance is enhanced in terms of bit error rate The Statistical based comparison is made between the two sensing techniques to evaluate the performance in terms of SNR $[5,6]$.

The rest part of the paper is systematized as follows: in section II literature survey is presented, Section III contains model for Energy detection, Cyclostationary detection, Section IV contains Results and Simulations and Section V contains Conclusion.

\section{LITERATURE SURVEY}

Partha Pratim Bhattacharya, et al., [6], presented an overview of all spectrum sensing techniques for cognitive radio. Various related aspects regarding each technique were studied properly. In addition to this the Cooperative sensing, External sensing and Distributed sensing were also described. Pros and cons of each technique give the overview to prefer one sensing technique over the other. Although The Basic comparison between the different sensing techniques was also given in this paper.

G. Mohapatra, et al., [5], Spectrum demonstrated that sensing problem has gained new aspects with cognitive radio and opportunistic spectrum access approach. To identify the unused spectrum is one of the most challenging issues in cognitive radio systems. This paper explored various sensing methods, 
Analyze their performance under certain conditions , applicability, effectiveness under different transmission conditions and advantages and disadvantages incorporated with each sensing method. This paper evaluated the performance of cognitive radio with energy detection based spectrum sensing (ED-SS) in AWGN, and Cyclostationary feature is used for Spectrum sensing. The results obtained by different windowing techniques were implemented along with their contour plots and a comparative study based on the simulation results was also given successfully. Cyclostationary technique based on window functions helped in detecting the primary user under low SNR condition.

Shiyu Xu, et al., [7], addressed the Cognitive radio in terms of Real time spectrum sensing with certain accuracy The Spectrum sensing technique used was Cyclostationary. This paper detected the licensed users' features by examining the performance of some frequencies. And Cycle frequencies which reduce the complexity significantly. Through simulation examination on different detection points, he finds out the best detection point. Combination detection method using multiple detection points to is used tonobtain better performance. The effectiveness of the proposed method was validated through results.

Abdullah Al-Mamun, et al., [2], This Paper examined how cognitive radio can be utilized in short range systems based on Ultra-Wideband (UWB). UWB is a technology in wireless communication. it is used for high speed data transmission with low power utilization with applications in many fields like military, radar, sensor, tracking, data collection or even commercial application. UWB can move between very low data rate or very high data rate and also among short range and long range distance applications. Impulse radio UWB showed some unique characteristics in short-range communications with varieties of throughput options that include high data rates. The strong cooperation between the aims of cognitive radio and features of IR-UWB has been shown in this paper.

\section{SYSTEM MODEL}

Two sensing techniques are being carried out for which model will be given

\subsection{Cyclostationary Detection}

This process basically depends on periodicity or statistics of mean or autocorrelation of the signal that vary periodically over time [7]. This periodicity is used in received signal to represent the existence of the primary users. The cyclic correlation factor (SCF) is the key point for detecting the primary user signal. The flow Chart of Cuclostationary detection technique is given as

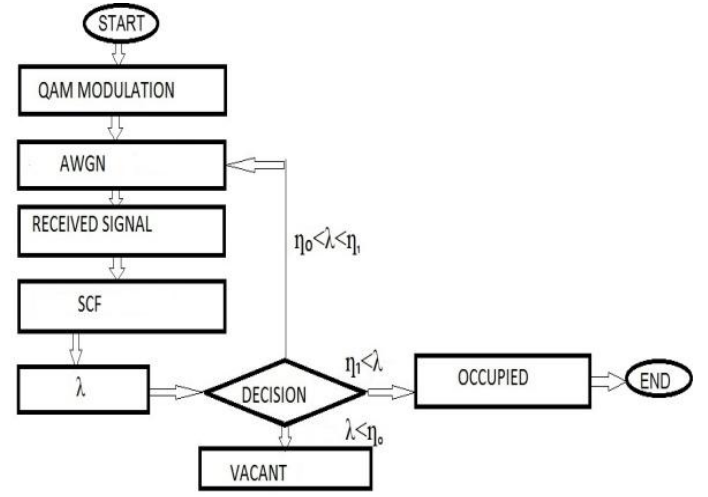

Figure 1. Flow Chart of Cyclostationary Detection

Initially we go for a check to evaluate the occupancy of the channel to show whether the user's data is present in its frequency band or it is empty; known as spectrum hole [8]. On the arrival of user data, it is modulated at the carrier frequency. Then all modulated signals are added to construct carrier signal. Periodogram is used for the estimation of power spectral density(PSD). Assignment of primary user with spectrum is done according to their data requirement. The first spectral hole is assigned to new secondary user. The particular slot is emptied if all the slots are filled. The effect of noise along with the attenuation is taken. To decide the behaviour of the signal whether it is periodic or aperiodic, spectral correlation function (SCF) along with different windows is used. Kaiser, Hamming, Hann and Rectangular windows that are used to decide the behaviour of signal. Due to the periodicity of the baseband signal, SCF would be capable to detect the primary user signal at very low SNR because of its noise rejection capability. This is because noise is totally random and does not exhibit any periodicity. The flow chart for the cyclostationary detection is given as:

The system model for Cyclostationary detection can be explained as follows. The conditions for the process to be cyclostationary:

$$
\left.\mathrm{E}\left\{\mathrm{x}+\mathrm{T}_{\mathrm{o}}\right)\right\}=\{(\mathrm{X}(\mathrm{t})\}
$$

A process $x(t)$ is said to be second-order cyclostationary in the wide sense if its mean and autocorrelation function are periodic with some period $\mathrm{T}>0$. Both the mean and auto correlation function needs to be periodic. Taking the Fourier coefficient, it is represented in terms of cyclic correlation function given as:

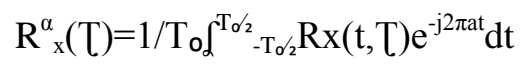

Where $\alpha=n / T_{n}$ represents the cyclic frequencies. Then, the discrete Fourier transformation of the cyclic autocorrelation function (CAF) can be computed to obtain the spectral correlation function (SCF) as:

$$
S(f, a)=\int_{-\infty}^{\infty} R_{x}^{\alpha}(T) e^{-j 2 \pi f t} d t
$$

Finally, the detection is completed by searching for the unique cyclic frequency corresponding to the peak in the SCF plane. The peak will not be present in the case when there is no primary user signal present in the concerned frequency range. By counting the number of secondary peaks at the double frequencies SCF can find out the type of modulation scheme used by the primary user signal. If the modulation scheme involved is BPSK, there will be single secondary peaks at the double of operating frequency. Instead if the modulation scheme involved is QPSK, there will be two such secondary peaks at the double of operating frequency.

\subsection{Energy Detection}

This is a most common method of spectrum sensing because of low complexity and computational cost. The receivers do not require any prior knowledge on the primary user's signal [9]. The system model for Energy detection is given as:-

The energy of an averaged signal subjected to two hypothetical test function.

\section{$\mathrm{H}_{1}$ Under $\mathrm{H}_{0}$}

Under $\mathrm{H}_{0} \mathrm{X}[\mathrm{n}]=\mathrm{W}[\mathrm{n}]$ : occurrence of noise signal

Under $\mathrm{H}_{1} \mathrm{X}[\mathrm{n}]=\mathrm{S}[\mathrm{n}]+\mathrm{W}[\mathrm{n}]$ : occurrence of signal with noise.

Here $\mathrm{n}=0,1,2 \ldots, \mathrm{N}-1, \mathrm{~N}$ shows the index of sample, $\mathrm{w}[\mathrm{n}]$ designate the noise and $s[n]$ is the primary signal to detect. 
The flow chart of Energy detection is :-

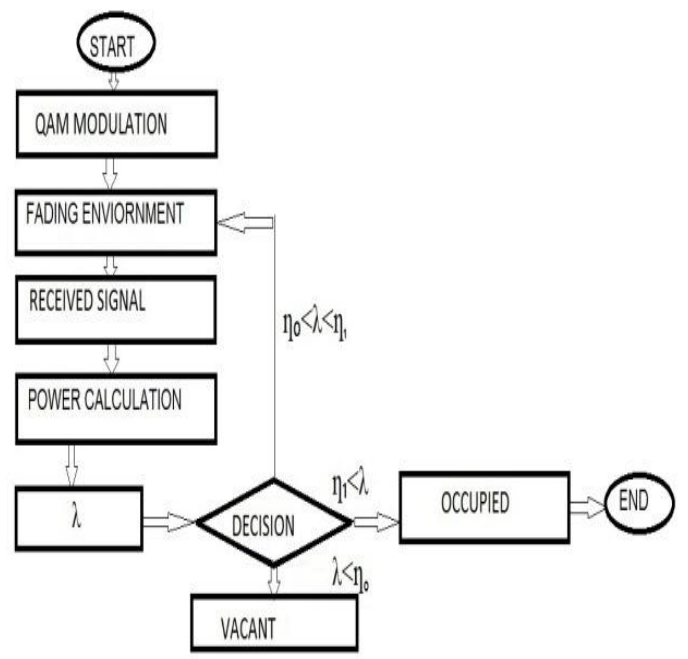

Figure 2. Flow Chart of Energy Detection

According to it, we can measure the energy of received signal by squaring the output of band pass filter and integrated over the observation time. Finally the output of the integrator $\mathrm{Y}$ is compared with threshold $\lambda$ to decide whether primary user is present or not. In the proposed work, threshold used is Chi square distribution.

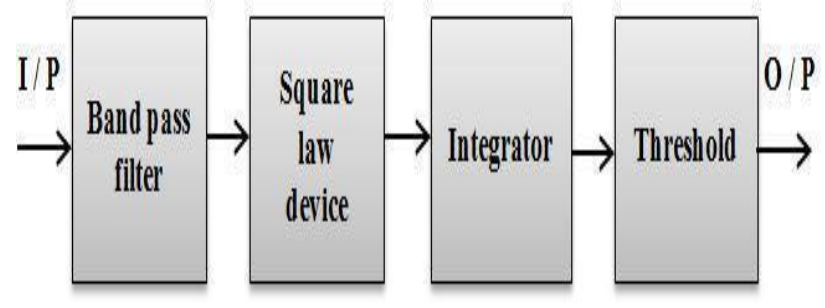

Figure 3. Block Diagram of Energy Detection

The Energy Detector can be applied to different fading channel which includes Rayleigh fading channel, Rician fading channel and MIMO fading channel to calculate the probability of detection $\mathrm{P}_{\mathrm{d}}$ and probability of false alarm $\mathrm{P}_{\mathrm{f}}$ for different channels as follows [10]:

$\mathrm{Pf}=\mathrm{P}\left\{\mathrm{Y}>{ }_{\mathrm{H}_{0}}\right\}=\mathrm{Q}_{\mathrm{m}}(\sqrt{ } 2 \lambda, \sqrt{ } \lambda)$

Where $\Gamma(),. \Gamma(.,$.$) are complete and incomplete gamma$ functions, $\gamma$ is SNR and $\mathrm{Q}_{\mathrm{m}}($ ) is the Marcum Q- function

$$
\mathrm{Pd}=\mathrm{P}\left\{\mathrm{Y}>^{\lambda /}{ }_{\mathrm{H}_{1}}\right\}=\left\{{ }^{\mathrm{r}(\mathrm{n}, \lambda 2)} / \Gamma(\mathrm{n})\right.
$$

\subsection{MIMO Fading Enviornment}

We simulate Energy detector over MIMO fading because Bit error rate (BER) is significantly enhanced at low signal to noise ratio (SNR). That means as we increase the number of antennas at receiver end, the BER is decreased 2 times thus providing higher capacity, better transmission quality, increased coverage area, low probability of false alarm $\mathrm{P}_{\mathrm{f}}$ and high probability of detection $\mathrm{P}_{\mathrm{d}}$. Moreover MIMO fading channel models other fading channels such as Rician and Rayleigh fading channel. The probability of detection $P_{d}$ and probability of False alarm $P_{f}$ for MIMO fading channel is given as:

$$
\mathrm{Pf}=\Gamma\left[\mathrm{L}, \mathrm{n} /\|\mathrm{h}\|^{2} \sigma^{2} \mathrm{n}\right] / \Gamma(\mathrm{L}) \quad \text { Where } \Gamma\left(\mathrm{L}, \mathrm{n} /\|\mathrm{h}\| \|^{2} \sigma^{2} \mathrm{n}\right)
$$

is upper incomplete gamma function and $\Gamma(\mathrm{L})$ is complete gamma function. $\mathrm{L}$ is the number of samples which each antenna receives and $\sigma_{\mathrm{n},} \sigma_{\mathrm{m}}$ are the noise variance and signal variance respectively. $\mathrm{H}$ is decision threshold.

$$
\mathrm{Pd}=\Gamma\left(\mathrm{L}, \mathrm{n} /\|\mathrm{h}\|^{2}\left(\|\mathrm{~h}\|^{2}{ }^{2}{ }_{\mathrm{s}}+\sigma_{\mathrm{n}}^{2} / \Gamma(\mathrm{L})\right.\right.
$$

\section{RESULTS AND CONCLUSIONS}

An extensive set of simulations have been conducted in MATLAB using the system model as described in the previous section. Research work of this paper is accomplished in such a manner that outcome will be a selection of highly optimized parametric algorithm which would help to evaluate effective spectrum sensing technique.

\subsection{Cyclostationary Detection based on HANN and Kaiser Window}

The behaviour of the periodicity of signal is determined by SCF using different windows such as Hann Window, Kaiser Window, and Blackman Harris Window so as to detect primary user low SNR. Peak in the graph shows the presence of primary user. In addition to this, the SCF can be used to find out the type of modulation scheme used by the primary user signal. Type of modulation scheme can be achieved by counting the number of secondary peaks at the double frequencies. If the modulation scheme involved is BPSK, there will be single secondary peaks at the double of operating frequency. Instead if the modulation scheme involved is QPSK, there will be two such secondary peaks at the double of operating frequency.

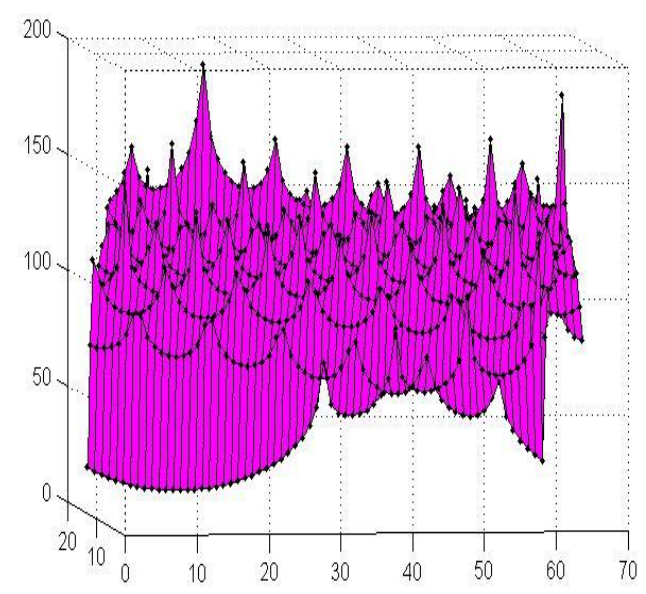

Figure 4. Detecting Primary user using Hanning window

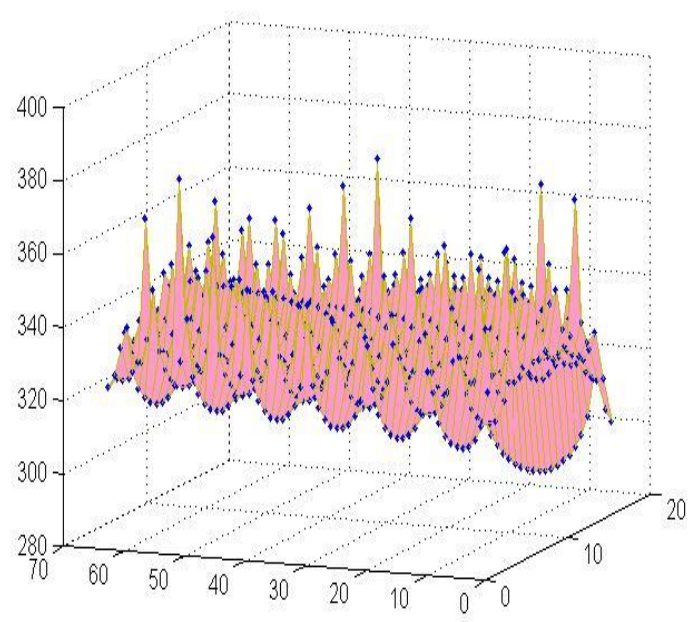

Figure 5. Detecting Primary user using Kaiser Window 


\subsection{Energy based Detector}

We use receiver characteristics (ROC) analysis for the signal detection theory to study the performance of the energy detector. We simulate the Energy detector over MIMO fading channel as it models both Rician, Rayleigh fading channel. A graph is plotted for probability of detection over MIMO, Rician, Rayleigh fading channels shown in the figure. The graph shows that with increasing probability of detection, there is increase in the probability of false alarm.

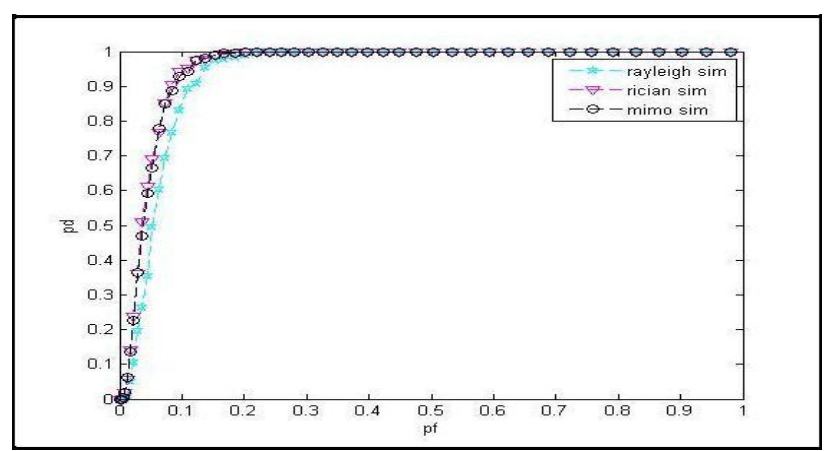

Figure 6. Analysis of pd with different fading channel for energy based detection

Lastly the Statistical comparison is made between the two to evaluate the performance in terms of SNR. From the graph, it is evident that Cyclo stationary performs better than the Energy detector because of its detection capability.

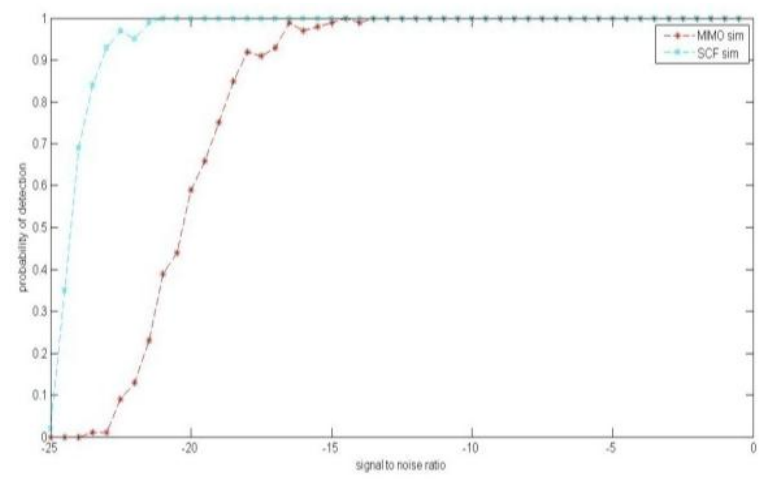

Figure 7. Comparison of Energy based and Cyclostationary based Detection

\section{CONCLUSION}

This paper takes the difficulty of ineffective use of spectrum given by FCC that spectrum is sufficient but is not properly utilized thus maximizing the utilization of the spectrum. In this paper, two Spectrum sensing techniques are successfully implemented. In Cyclostationary technique, the PSD is successfully introduced to check the proper allocation of the spectrum. Noise along with attenuation is considered on the signal efficiently. Spectral correlation function with different windows is used well to calculate the behaviour of the signal whether it is periodic or aperiodic . Based on periodicity of the baseband signal, primary user signal at low signal to noise ratio is computed successfully. Moreover Energy Detector over MIMO fading is developed positively that increases performance in term of BER, increases capacity, provides better transmission quality. Finally the comparison between the two is made successfully to evaluate the performance in terms of SNR which shows that Cyclostationary detects well as compared to Energy detection.

\section{REFERENCES}

[1] G. Q Maguire and J. Mitola, "Cognitive radio: Making Software Radio more Personal", IEEE Personal Communication Magazine, vol. 6, no. 4, (1999) August, pp. 13-18.

[2] A. Al-Mamun and M. Rafiq Ullah, "Cognitive Radio for Short Range Systems based on Ultra-Wide Band", in Department of Signal Processing, Blekinge Institute of Technology, (2011).

[3] S. Kapoor and G. Singh, "Non Cooperative Spectrum Sensing: A Hybrid Model Approach", IEEE International Conference on Devices and Communication, Mesra, 24-25 Feb,2011, pp. 1-5.

[4] Ambarish G. Mohapatra, Dr. S. K. Lenka, Subhashri G. Mohapatra, "Performance evaluation of cyclostationary based spectrum sensing in cognitive radio network," IEEE International Multi Conference on Automation, Computing, Communication, Control and Compressed Sensing, Kottayam, 2013, pp. 90-97.

[5] Anita Garhwal and Partha Pratim Bhattacharya, "A Survey on Spectrum Sensing Techniques in Cognitive Radio," International Journal of Computer Science \& Communication Networks , vol. 1(2), pp. 196-206, 2011.

[6] Zhijin Zhao, Junna Shang, Shiyu Xu, "Spectrum Sensing Based on Cyclostationarity," Workshop on Power Electronics and Intelligent Transportation System,IEEE, Guangzhou , 2-3 Aug 2008, pp. 171-174.

[7] Haider M. Al Sabbagh, Ahmed S. Kadhim, "Detection the Spectrum Holes in the Primary Bandwidth of the Cognitive Radio Systems in Presence Noise and Attenuation," Int. J. Communications, Network and System Sciences, , pp. 684-690, 2012.

[8] R.Saravanan, R.Muthaiah, M.Lakshmi, "Energy Detection Based Spectrum sensing for cognitive Radio," International Journal of Engineering and Technology, vol. 5, no. 2, pp. 963-967, Apr-May 2013.

[9] Shakya Sudeep, Koirala Nirajan,Nepal Narayan, "Energy detection based techniques for Spectrum sensing in Cognitive radio over different fading channels," Journal of Selected Areas in Telecommunication (JSAT), pp. 15-19.

[10] D. Niyato, Z. Han and E. Hossian, "Dynamic Spectrum Access and Management in Cognitive Radio Networks", Cambridge University Press., (2009) July. 